\title{
Tevatron anomalies and LHC cross-checks
}

\author{
Germán Rodrigo*i \\ Instituto de Física Corpuscular, \\ Consejo Superior de Investigaciones Científicas-Universitat de València, \\ Parc Científic, E-46980 Paterna (Valencia), Spain. \\ E-mail: german.rodrigo@csic.es
}

\begin{abstract}
A sizeable charge asymmetry in top quark pair production has been observed at the Tevatron. The experimental results seem to exceed systematically the Standard Model theory predictions by a significant amount and have triggered a large number of suggestions for 'new physics'. The effect is also visible at the LHC, and preliminary results have already been presented by the ATLAS and CMS collaborations. In this talk, we review the present status of the theoretical predictions, and their comparison with the experimental measurements.
\end{abstract}

The 2011 Europhysics Conference on High Energy Physics-HEP 2011,

July 21-27, 2011

Grenoble, Rhône-Alpes, France

*Speaker.

†Supported by REA Grant Agreement PITN-GA-2010-264564 (LHCPhenoNet), by the MICINN Grant No. FPA2007-60323, and PR2010-0481, by CPAN (Grant No. CSD2007-00042), and by the Generalitat Valenciana Grant No. PROMETEO/2008/069. G.R. acknowledges hospitality at the Institut für Theoretische Teilchenphysik of the Karlsruher Institut für Technologie during the completion of this work, and J. H. Kühn for a fruitful collaboration. 


\section{Introduction}

Top quark production at hadron colliders is one of the most active fields of current theoretical and experimental studies [1], and most probably the most promising probe of physics beyond the Standard Model (SM). Sizable differences have been observed between theory predictions $[2,3,4,5]$ for the top quark charge asymmetry and measurements by the CDF and the D0 collaborations $[6,7,8,9]$ at the Tevatron. The discrepancy is particularly pronounced for the subsample of $t \bar{t}$ pairs with large invariant mass, $m_{t \bar{t}}>450 \mathrm{GeV}$, and the asymmetry defined in the $t \bar{t}$ rest frame, where a $3.4 \sigma$ effect has been claimed [8]. It is interesting to note, however, that the discrepancy is less prominent in the laboratory frame [8]. The D0 Collaboration also finds positive discrepancies with the SM [6]. These discrepancies have triggered a large number of theoretical investigations, using these results, either to restrict new physics like heavy axigluons [10,11] or to postulate a variety of new phenomena in the t-channel $[12,13,14]$. At the same time, the robustness of the leading order QCD prediction has been studied in $[15,16]$, where it has been argued that next-to-leading (NLL) as well as next-to-next-to leading (NNLL) logarithmic corrections do not significantly modify the leading order result, in agreement with the approach advocated in [3,4] (Note, however, the large corrections observed in Ref. [17] for the corresponding studies of the $t \bar{t}+$ jet sample). A small modification of the SM prediction arises from inclusion of QED corrections. In Ref. [3] this effect was estimated to lead to an increase of the the QCD asymmetry by a factor 1.09, in recent analysis [18, 19], however, an enhancement factor of 1.2 has been obtained. Obviously this small increase of the SM prediction for the asymmetry cannot resolve the discrepancy between theory an experiment mentioned above.

In this talk we revisit the SM prediction of the top quark charge asymmetry at the Tevatron and the LHC [18]. We paid special attention to the electroweak corrections. We summarize the experimental measurements of the asymmetry and update the pull of their discrepancy with the SM. We also analyze the effect of introducing a cut in the $t \bar{t}$ transverse momentum (see also [20]). Finally, we introduce a new quantity $A_{t \bar{t}}(Y)$, which measures the forward-backward asymmetry with respect to the average rapidity of top and antitop quarks, being a suitable observable both at the Tevatron and the LHC. Beyond the SM contributions to the asymmetry have also been discussed in this conference in [21], and will not be covered in this document.

\section{The charge asymmetry in the $S M$}

The dominant contribution to the charge asymmetry originates from $q \bar{q}$ annihilation $[3,4]$. Specifically, it originates from the interference between the Born amplitudes for $q \bar{q} \rightarrow Q \bar{Q}$ and the part of the one-loop correction, which is antisymmetric under the exchange of quark and antiquark (box and crossed box). To compensate the infrared divergences, this virtual correction must be combined with the interference between initial and final state radiation. Diagrams with triple gluon coupling in both real and virtual corrections give rise to symmetric amplitudes and can be ignored. The corresponding contribution to the rate is conveniently expressed by the absorptive contributions (cuts) of the diagrams depicted in Fig 1. A second contribution to the asymmetry from quark-gluon scattering ("flavor excitation") hardly contributes to the asymmetry at the Tevatron. At the LHC, it enhances the asymmetry in suitable chosen kinematical regions [4]. 


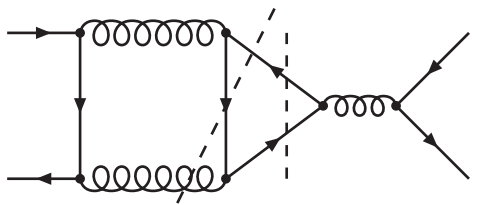

(a)

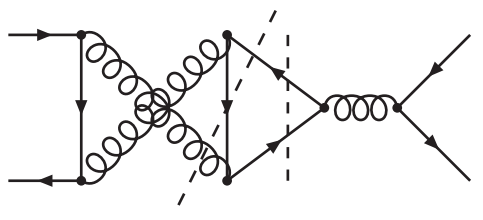

(b)

Figure 1: Cut diagrams representing the QCD contribution to the charge asymmetry.

Diagrams similar to those depicted in Fig. 1, where one of the gluons has been substituted by a photon, also lead to a contribution to the charge asymmetry from mixed QED-QCD corrections. The relative factor between QCD and QED asymmetries amounts to

$$
f_{q}^{\mathrm{QED}}=3 \frac{\alpha_{\mathrm{QED}} Q_{t} Q_{q}}{\frac{\alpha_{S}}{2}\left(\frac{d_{a b c}^{2}}{4}\right)^{2}}=\frac{\alpha_{\mathrm{QED}}}{\alpha_{S}} \frac{36}{5} Q_{t} Q_{q}
$$

for one quark species, and to

$$
f^{\mathrm{QED}}=\frac{4 f_{u}^{\mathrm{QED}}+f_{d}^{\mathrm{QED}}}{5}=\frac{\alpha_{\mathrm{QED}}}{\alpha_{S}} \frac{56}{25} \approx 0.18
$$

after convolution with the PDFs if one considers as a first approximation that the relative importance of $u \bar{u}$ versus $d \bar{d}$ annihilation at the Tevatron is $4: 1$. Thus, to an enhancement of nearly twenty percent of the QCD asymmetry, in good agreement with the more detailed numerical studies of $[18,19]$. At the LHC, the relative importance of $u \bar{u}$ versus $d \bar{d}$ annihilation is approximately $2: 1$, thus reducing $f^{\text {QED }}$ by a factor $5 / 7$ down to 0.13 . Similarly, weak contributions with the photon replaced by the $Z$ boson should be considered at the same footing. However, as a consequence of the cancellation between up and down quark contributions, and the smallness of the weak coupling, the weak corrections at the Tevatron are smaller by more than a factor 10 than the corresponding QED result. For proton-proton collisions the cancellation between up and down quark contributions is even stronger and the total weak correction is completely negligible.

\section{Tevatron}

Assuming that the rapidities of $t$ and $\bar{t}$ have been measured simultaneously, one defines the asymmetry

$$
A_{t \bar{t}}(Y)=\frac{N\left(y_{t}>y_{\bar{t}}\right)-N\left(y_{\bar{t}}>y_{t}\right)}{N\left(y_{t}>y_{\bar{t}}\right)+N\left(y_{\bar{t}}>y_{t}\right)},
$$

where $Y=\left(y_{t}+y_{\bar{t}}\right) / 2$ has been fixed. An almost flat asymmetry $A_{t \bar{t}}(Y)$ of around $8 \%$ is predicted at Tevatron as a function of $Y$ (Fig. 2 left). Two versions of the integrated asymmetry have been introduced in Refs. [2, 3, 4]: the forward-backward asymmetry in the laboratory frame

$$
A_{\text {lab }}=\frac{N\left(y_{t}>0\right)-N\left(y_{t}<0\right)}{N\left(y_{t}>0\right)+N\left(y_{t}<0\right)}=\frac{N\left(y_{t}>0\right)-N\left(y_{\bar{t}}>0\right)}{N\left(y_{t}>0\right)+N\left(y_{\bar{t}}>0\right)},
$$



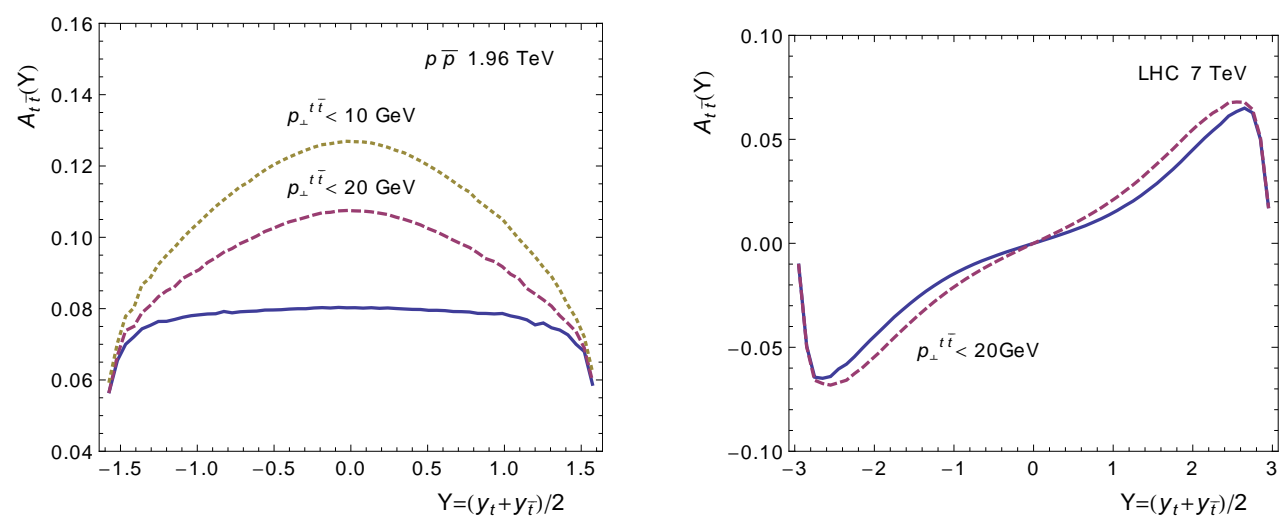

Figure 2: Pair charge asymmetry $A_{t \bar{t}}(Y)$ as a function of the mean rapidity $Y=\left(y_{t}+y_{\bar{t}}\right) / 2$. Solid line: without cut on $p_{\perp}^{t \bar{t}}$, dotted/dashed lines: with cut on $p_{\perp}^{t \bar{t}}$.

Table 1: Predicted asymmetries in the laboratory $A_{\text {lab }}$ and the $t \bar{t}$ rest frame $A_{t \bar{t}}$ at Tevatron. Predictions are given also for samples with the top quark pair invariant mass $m_{t \bar{t}}$ above and below $450 \mathrm{GeV}$, and with $|\Delta y|=\left|y_{t}-y_{\bar{t}}\right|$ larger or smaller than one in the $t \bar{t}$ rest frame.

\begin{tabular}{|c|ccccc|}
\hline laboratory & $A_{\text {lab }}$ & $m_{t \bar{t}}<450 \mathrm{GeV}$ & $m_{t \bar{t}}>450 \mathrm{GeV}$ & & \\
\hline SM & $0.056(7)$ & $0.029(2)$ & $0.102(9)$ & & \\
\hline MCFM [8] & $0.038(6)$ & & & & \\
\hline \hline$t \bar{t}$ rest frame & $A_{t \bar{t}}$ & $m_{t \bar{t}}<450 \mathrm{GeV}$ & $m_{t \bar{t}}>450 \mathrm{GeV}$ & $|\Delta y|<1$ & $|\Delta y|>1$ \\
\hline SM & $0.087(10)$ & $0.062(4)$ & $0.128(11)$ & $0.057(4)$ & $0.193(15)$ \\
\hline MCFM [8] & $0.058(9)$ & $0.040(6)$ & $0.088(13)$ & $0.039(6)$ & $0.123(18)$ \\
\hline
\end{tabular}

and the asymmetry in the $t \bar{t}$ rest frame

$$
A_{t \bar{t}}=\frac{N\left(y_{t}>y_{\bar{t}}\right)-N\left(y_{\bar{t}}>y_{t}\right)}{N\left(y_{t}>y_{\bar{t}}\right)+N\left(y_{\bar{t}}>y_{t}\right)} .
$$

Results for both of them in the SM are listed in Table 1. These predictions include also the QED and weak (strongly suppressed) corrections. Those corrections enhance the QCD asymmetry by an overall factor 1.21, which is slightly different from Eq. (2.2) due to the deviation of the relative amount of $u \bar{u}$ and $d \bar{d}$ contributions from the simple approximation $4: 1$.

In order to compare theoretical results in the SM with the most recent measurements at Tevatron, predictions in Table 1 are presented also for samples with $m_{t \bar{t}}$ larger and smaller than $450 \mathrm{GeV}$, and with $|\Delta y|=\left|y_{t}-y_{\bar{t}}\right|$ larger and smaller than 1. It is also interesting to compare these results with those based on a Monte Carlo prediction [8] based on MCFM [22]. The enhancement factor of the SM result in Table 1 compared to MCFM of about 1.5 is easily understood: a factor 1.2 originates from the inclusion of QED effects. Another factor of about 1.3 originates from normalizing with respect to the Born cross-section instead of the NLO result. Since the asymmetric part of the cross-section is presently known to LO only we consider the normalization to the LO cross-section more plausible $[3,4,15,16]$. 


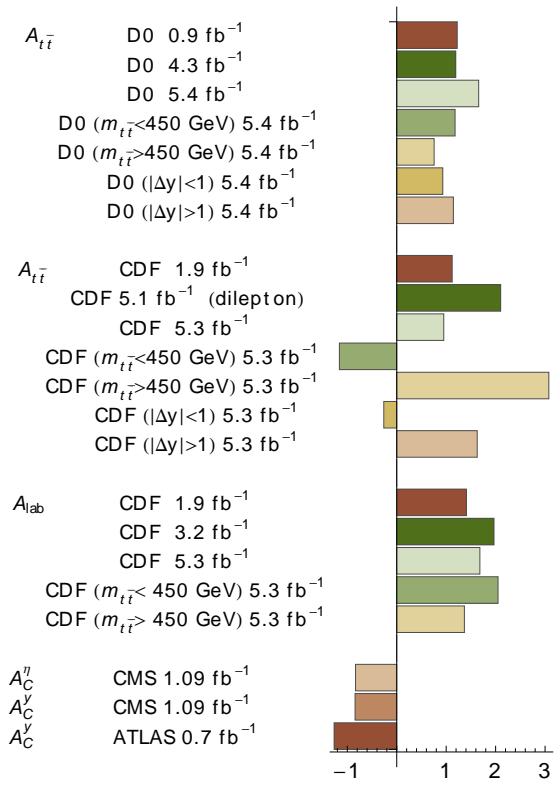

Figure 3: Summary of experimental measurements of the charge asymmetry in comparison with the SM theoretical predictions. The histogram represents the pull of the discrepancy for each measurement.

A graphical illustration of the results in terms of the "pull" (measured in standard deviations) is shown in Fig. 3. The systematic upward shift of all but two Tevatron results is evident. The highest discrepancy, as has extensively been discussed in the literature, occurs for samples with $m_{t \bar{t}}>450 \mathrm{GeV}$ and the charge asymmetry defined in the $t \bar{t}$ rest frame. Also shown in this Figure are preliminary results from CMS [23] and ATLAS [24] with a slight pull in the opposite direction.

The impact of cuts on hard gluon (and photon) radiation on $A_{t \bar{t}}(Y)$ is also shown in Fig. 2. The dotted and dashed curves in Fig. 2 show the effect of a cut on $p_{\perp}^{t \bar{t}}$ for values of $p_{\perp}^{\max }=10 \mathrm{GeV}$ and $20 \mathrm{GeV}$, respectively. An increase of the asymmetry by more than a factor 1.5 in the central region is observed for the most restrictive choice of $10 \mathrm{GeV}$, and even a fairly loose $p_{\perp}^{\max }=20 \mathrm{GeV}$ modifies the asymmetry by up to a factor 1.3 .

\section{LHC}

The charge asymmetry can also be investigated in proton-proton collisions at the LHC $[2,3,4]$ by exploiting the small $t \bar{t}$ sample produced in annihilation of valence quarks and antiquarks from the sea. Since valence quarks carry on average more momentum than sea antiquarks, production of top quarks with larger rapidities will be preferred in the SM, and antitop quarks will be produced more frequently at smaller rapidities. This observation suggests to define the cut-dependent asymmetries

$$
A_{C}^{\text {in }}\left(y_{C}\right)=\frac{N\left(\left|y_{\bar{t}}\right| \leq y_{C}\right)-N\left(\left|y_{t}\right| \leq y_{C}\right)}{N\left(\left|y_{t}\right| \leq y_{C}\right)+N\left(\left|y_{\bar{t}}\right| \leq y_{C}\right)} \quad \text { and } \quad A_{C}^{\text {out }}\left(y_{C}\right)=\frac{N\left(\left|y_{t}\right|>y_{C}\right)-N\left(\left|y_{\bar{t}}\right|>y_{C}\right)}{N\left(\left|y_{t}\right|>y_{C}\right)+N\left(\left|y_{\bar{t}}\right|>y_{C}\right)},
$$

which serve to characterize the depletion of top quarks in the central region $\left(A_{C}^{\text {in }}\left(y_{C}\right)>A_{C}^{\text {out }}\left(y_{C}\right)\right.$ for $y_{C} \lesssim 0.7$ approximately $\left.[2,25,26]\right)$, and their enhancement at larger rapidities; $A_{C}^{\text {out }}$ is much larger 
Table 2: SM cut-independent charge asymmetries $A_{\eta}$ and $A_{y}$, and integrated pair charge asymmetry $A_{t \bar{t}}^{\text {cut }}\left(Y_{\text {cut }}=0.7\right)$, at different LHC energies. Summary of recent measurements by CMS and ATLAS.

\begin{tabular}{|l|ccc|}
\hline & $A_{C}^{\eta}$ & $A_{C}^{y}$ & $A_{t \bar{t}}^{\text {cut }}\left(Y_{\text {cut }}=0.7\right)$ \\
\hline LHC 7 TeV & $0.0136(8)$ & $0.0115(6)$ & $0.0203(8)$ \\
LHC 14 TeV & $0.0077(4)$ & $0.0059(3)$ & $0.0100(4)$ \\
\hline \hline LHC 7 TeV CMS [23] & $-0.016 \pm 0.030_{-0.019}^{+0.010}$ & $-0.013 \pm 0.026_{-0.021}^{+0.026}$ & \\
LHC 7 TeV ATLAS [24] & & $-0.024 \pm 0.016 \pm 0.023$ & \\
\hline
\end{tabular}

than $A_{C}^{\text {in }}$ at large values of the rapidity cut $y_{C}$ [18]. This is because the central region is dominated by gluon fusion processes, while the sample with large rapidities has a larger relative content of $q \bar{q}$ initiated events. The statistical significance of both observables is, however, very similar [27] because the larger size of the asymmetry $A_{C}^{\text {out }}$ with respect to $A_{C}^{\text {in }}$ is compensated by the lower rate of events at larger rapidities.

The recent CMS [23] and ALTAS [24] analysis have considered also the cut-independent charge asymmetries

$$
A_{C}^{\eta}=\frac{N\left(\Delta_{\eta}>0\right)-N\left(\Delta_{\eta}<0\right)}{N\left(\Delta_{\eta}>0\right)+N\left(\Delta_{\eta}<0\right)} \quad \text { and } \quad A_{C}^{y}=\frac{N\left(\Delta_{y}>0\right)-N\left(\Delta_{y}<0\right)}{N\left(\Delta_{y}>0\right)+N\left(\Delta_{y}<0\right)}
$$

where $\Delta_{\eta}=\left|\eta_{t}\right|-\left|\eta_{\bar{t}}\right|$ and $\Delta_{y}=\left|y_{t}\right|-\left|y_{\bar{t}}\right|$ or $y_{t}^{2}-y_{\bar{t}}^{2}$. The SM predictions for the integrated asymmetries are listed Table 2 for different center-of-mass energies of the LHC, together with the experimental results for $\sqrt{s}=7 \mathrm{TeV}$. Both experiments obtain negative asymmetries, although compatible with the SM prediction within uncertainties. New analysis with larger statistics are underway.

Top quark production in proton-proton collisions is dominated by gluon fusion, which, in turn, is dominant in the central region. Conversely, quark-antiquark annihilation will be more enriched for events with $t \bar{t}$ at larger rapidities (and larger $m_{t \bar{t}}$ ). This suggest to employ the definition of Eq. (3.1), which is essentially the asymmetry in the $t \bar{t}$ rest frame, also for the present case, and concentrate on $t \bar{t}$ events at large rapidities. The prediction for $A_{t \bar{t}}(Y)$ is shown in Fig. 2 for $\sqrt{s}=7 \mathrm{TeV}$ (right plot). By construction, $A_{t \bar{t}}(Y)$ is now an antisymmetric function of $Y$. Since most of the charge asymmetry is concentrated at large rapidities the statistical significance of any measurement will be enhanced, if the sample is restricted to larger rapidities. Let us therefore define the quantity

$$
A_{t \bar{t}}^{\mathrm{cut}}\left(Y_{\mathrm{cut}}\right)=\frac{N\left(y_{t}>y_{\bar{t}}\right)-N\left(y_{\bar{t}}>y_{t}\right)}{N\left(y_{t}>y_{\bar{t}}\right)+N\left(y_{\bar{t}}>y_{t}\right)}
$$

where $Y>Y_{\text {cut }}$. Theoretical predictions in the SM for $A_{t \bar{t}}^{\text {cut }}\left(Y_{\text {cut }}=0.7\right)$ are presented in Table 2. QED and weak corrections amount to roughly a factor 1.1 .

\section{Summary}

Tevatron has shown in the last years a systematic upward discrepancy in the measurement of the top quark charge asymmetry with respect to theoretical predictions in the SM. These discrepancies have triggered a large number of theoretical speculations about possible contributions beyond 
the SM. The Tevatron collaborations can still increase the statistical significance of their measurements, particularly by combining CDF and D0 results. On the other hand, the LHC, due to his present good performance, will provide quite soon competitive and accurate measurements of this effect.

\section{References}

[1] A. B. Galtieri et al. [ CDF and D0 Collaboration ], [arXiv:1109.2163 [hep-ex]].

[2] O. Antuñano, J. H. Kühn, G. Rodrigo, Phys. Rev. D77 (2008) 014003 [arXiv:0709.1652 [hep-ph]].

[3] J. H. Kühn, G. Rodrigo, Phys. Rev. D59 (1999) 054017 [hep-ph/9807420].

[4] J. H. Kühn, G. Rodrigo, Phys. Rev. Lett. 81 (1998) 49-52 [hep-ph/9802268].

[5] M. T. Bowen, S. D. Ellis, D. Rainwater, Phys. Rev. D73 (2006) 014008 [hep-ph/0509267].

[6] V. M. Abazov et al. [ D0 Collaboration ], [arXiv:1107.4995 [hep-ex]].

[7] V. M. Abazov et al. [D0 Collaboration], Phys. Rev. Lett. 100 (2008) 142002 [arXiv:0712.0851 [hep-ex]].

[8] T. Aaltonen et al. [ CDF Collaboration ], Phys. Rev. D83 (2011) 112003 [arXiv:1101.0034 [hep-ex]].

[9] T. Aaltonen et al. [CDF Collaboration], Phys. Rev. Lett. 101 (2008) 202001 [arXiv:0806.2472 [hep-ex]].

[10] P. Ferrario, G. Rodrigo, Phys. Rev. D80 (2009) 051701 [arXiv:0906.5541 [hep-ph]].

[11] G. Rodrigo, P. Ferrario, Nuovo Cim. C33 (2010) 04 [arXiv:1007.4328 [hep-ph]].

[12] S. Jung, H. Murayama, A. Pierce, J. D. Wells, Phys. Rev. D81 (2010) 015004 [arXiv:0907.4112 [hep-ph]].

[13] K. Cheung, W. -Y. Keung, T. -C. Yuan, Phys. Lett. $B 682$ (2009) 287-290 [arXiv:0908.2589 [hep-ph]]

[14] J. Shu, T. M. P. Tait, K. Wang, Phys. Rev. D81 (2010) 034012 [arXiv:0911.3237 [hep-ph]]

[15] L. G. Almeida, G. F. Sterman, W. Vogelsang, Phys. Rev. D78 (2008) 014008 [arXiv:0805.1885 [hep-ph]].

[16] V. Ahrens, A. Ferroglia, M. Neubert, B. D. Pecjak, L. L. Yang, JHEP 1009 (2010) 097 [arXiv:1003.5827 [hep-ph]].

[17] S. Dittmaier, P. Uwer, S. Weinzierl, Eur. Phys. J. C59 (2009) 625-646 [arXiv:0810.0452 [hep-ph]].

[18] J. H. Kühn and G. Rodrigo, to appear in JHEP, arXiv:1109.6830 [hep-ph].

[19] W. Hollik, D. Pagani, arXiv:1107.2606 [hep-ph].

[20] R. Demina, Presented at HEP-EPS Grenoble 2011.

[21] S. Westhoff, Presented at HEP-EPS Grenoble 2011, arXiv:1108.3341 [hep-ph].

[22] J. M. Campbell, R. K. Ellis, Phys. Rev. D60 (1999) 113006 [hep-ph/9905386].

[23] [ CMS Collaboration ], CMS-PAS-TOP-10-010, CMS-PAS-TOP-11-014.

[24] [ ATLAS Collaboration ], ATLAS-CONF-2011-106.

[25] P. Ferrario, G. Rodrigo, Phys. Rev. D78 (2008) 094018 [arXiv:0809.3354 [hep-ph]].

[26] P. Ferrario, G. Rodrigo, JHEP 1002 (2010) 051 [arXiv:0912.0687 [hep-ph]].

[27] J. L. Hewett, J. Shelton, M. Spannowsky, T. M. P. Tait, M. Takeuchi, arXiv:1103.4618 [hep-ph]. 